Marquette University
e-Publications@Marquette

Civil and Environmental Engineering Faculty

Research and Publications

Civil, Construction, and Environmental Engineering, Department of

2013

\title{
Influence of Fluid-Structure Interaction on Microcantilever Vibrations: Applications to Rheological Fluid Measurement and Chemical Detection
}

\author{
Isabelle Dufour \\ Université de Bordeaux \\ E. Lemaire \\ University of Bordeaux \\ B. Caillard \\ University of Bordeaux \\ H. Debeda \\ University of Bordeaux \\ C. Lucat \\ Université de Bordeaux
}

See next page for additional authors

Follow this and additional works at: https://epublications.marquette.edu/civengin_fac

Part of the Civil and Environmental Engineering Commons

\section{Recommended Citation}

Dufour, Isabelle; Lemaire, E.; Caillard, B.; Debeda, H.; Lucat, C.; Heinrich, Stephen M.; Josse, Fabien; and Brand, Oliver, "Influence of Fluid-Structure Interaction on Microcantilever Vibrations: Applications to Rheological Fluid Measurement and Chemical Detection" (2013). Civil and Environmental Engineering Faculty Research and Publications. 19.

https://epublications.marquette.edu/civengin_fac/19 


\section{Authors}

Isabelle Dufour, E. Lemaire, B. Caillard, H. Debeda, C. Lucat, Stephen M. Heinrich, Fabien Josse, and Oliver Brand 


\title{
Influence of fluid-structure interaction on microcantilever vibrations: applications to rheological fluid measurement and chemical detection
}

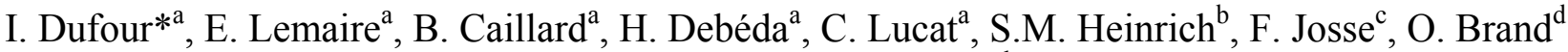 \\ ${ }^{a}$ Univ. Bordeaux, IMS, UMR 5218, F-33400 Talence, France; ${ }^{b}$ Department of Civil, Construction \\ and Environmental Engineering, Marquette University, Milwaukee, WI, USA; ${ }^{\mathrm{c}}$ Department of \\ Electrical and Computer, Marquette University, Milwaukee, WI, USA; ${ }^{\mathrm{d}}$ School of Electrical and \\ Computer Engineering, Georgia Institute of Technology, Atlanta, GA, USA
}

\begin{abstract}
At the microscale, cantilever vibrations depend not only on the microstructure's properties and geometry but also on the properties of the surrounding medium. In fact, when a microcantilever vibrates in a fluid, the fluid offers resistance to the motion of the beam. The study of the influence of the hydrodynamic force on the microcantilever's vibrational spectrum can be used to either (1) optimize the use of microcantilevers for chemical detection in liquid media or (2) extract the mechanical properties of the fluid. The classical method for application (1) in gas is to operate the microcantilever in the dynamic transverse bending mode for chemical detection. However, the performance of microcantilevers excited in this standard out-of-plane dynamic mode drastically decreases in viscous liquid media. When immersed in liquids, in order to limit the decrease of both the resonant frequency and the quality factor, alternative vibration modes that primarily shear the fluid (rather than involving motion normal to the fluid/beam interface) have been studied and tested: these include inplane vibration modes (lateral bending mode and elongation mode). For application (2), the classical method to measure the rheological properties of fluids is to use a rheometer. To overcome the limitations of this classical method, an alternative method based on the use of silicon microcantilevers is presented. The method, which is based on the use of analytical equations for the hydrodynamic force, permits the measurement of the complex shear modulus of viscoelastic fluids over a wide frequency range.
\end{abstract}

Keywords: Hydrodynamic force, microcantilever vibration, rheology, chemical detection, in-plane vibration, MEMS

\section{INTRODUCTION}

In recent years, interest in microcantilever-based chemical and bio-chemical sensing systems has risen due to their projected high sensitivity [1-5]. The large ratio of surface area to volume makes the microcantilever extremely sensitive to surface processes. For (bio)chemical detection, the microcantilever is usually coated with a (bio)chemically sensitive layer whose purpose is to selectively sorb the analyte or molecule of interest. The sorbed substance can then be detected by monitoring either the resonant frequency shift (dynamic mode) or the quasi-static deflection (static mode). A simplified way to explain the basic principle of such sensors is to say that in the case of dynamic mode operation, the change in mass associated with the sorption of analyte onto/into the sensitive coating causes a shift in resonant frequency, which may be correlated to the ambient concentration of the target substance. For static-mode operation, the sorption of analyte causes a cantilever deflection that is induced by surface stress or by the tendency of the sensitive coating to expand or contract upon analyte sorption or desorption (modification of strain and stress in the coating). In this paper we will focus on the use of the dynamic mode.

At the microscale, cantilever vibrations depend not only on the microstructure's properties and geometry but also on the properties of the surrounding medium (density and viscoelastic properties). In fact, when a microcantilever vibrates in a fluid, the fluid offers resistance to the motion of the beam. The coupling between the structure and the surrounding viscous fluid influences the structure's mechanical resonance; in particular, the fluid causes a decrease of both the resonant frequency and the quality factor and, thus, negatively impacts the device's sensing capabilities. In gas, resonant frequencies of classical transverse flexural modes may be reduced by a few percent compared with the value in vacuum, whereas the quality factor may exhibit reductions of two orders of magnitude. This performance degradation for conventional out-of-plane flexural resonant modes is further exacerbated in liquids: the resonant frequency and quality factor values are approximately one order of magnitude smaller than their values in the gas phase [6]. The reduced values 
of these resonance characteristics in liquid media adversely affect the sensitivity and the detection limit of these types of sensors.

In the present paper, we propose alternative uses of microcantilevers, including the implementation of unconventional vibrational modes to overcome the aforementioned obstacles, and demonstrate successful liquid-phase operation. By understanding in detail the influence of the hydrodynamic force on the microcantilever's vibrational spectrum, the following two objectives may be achieved: (1) optimization of microcantilevers for chemical detection in liquid media and (2) extraction of the mechanical properties of complex fluids.

The paper is organized such that a review of hydrodynamic force expressions and their impact on the resonant frequency and quality factor is given in Section 2 for the cases of out-of-plane and in-plane vibrations. In Section 3 we present how one may use the knowledge of the hydrodynamic force to choose appropriate vibrational modes and microcantilever geometries for chemical detection in liquid media. A second example of how a firm grasp of fluid-structure interaction may be used to advantage is the implementation of a microcantilever to extract the rheological properties of a fluid, which is described in Section 4.

Notation (Fig.1): The geometry of the cantilever is defined by the width $b$, thickness $h$, and length $L$. Coordinate $x$ is measured along the beam length. The properties of the cantilever material are the Young's modulus $E$ and the mass density $\rho$. The fluid (gas or liquid) properties are defined as $\rho_{f}$, the fluid's mass density, and $\eta$, the fluid's dynamic viscosity.

\section{FLUID-STRUCTURE INTERACTION: HYDRODYNAMIC FORCE AND MICROSTRUCTURE RESONANT CHARACTERISTICS}

In this paper, we focus on three distinct vibration modes (Fig.1): the transverse (out-of-plane) bending mode, the lateral (in-plane) bending mode and the elongation mode (also in-plane). A fourth mode that may also have interesting potential for both chemical detection and rheological sensing is the torsional mode, whose hydrodynamic force and resonant characteristics have been studied in [7-8]; however, that mode is not included in the present study.
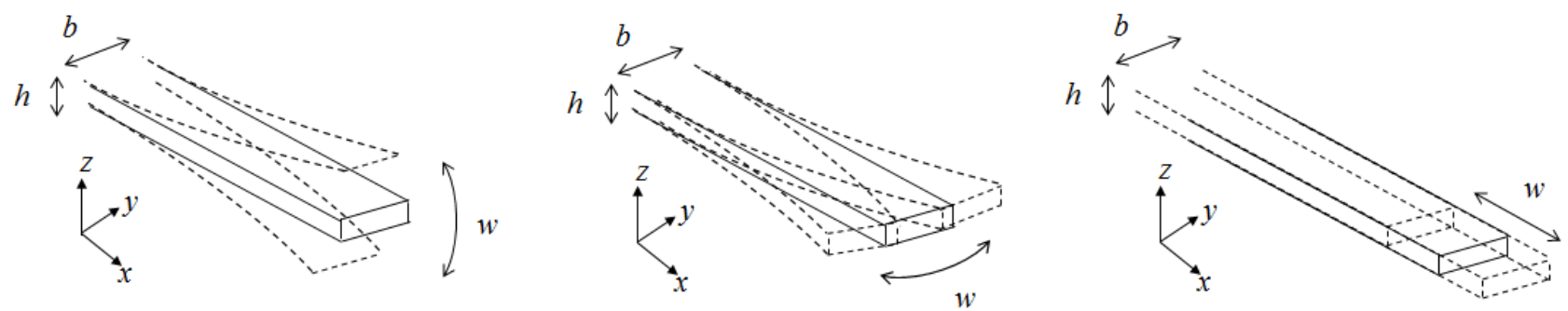

Figure 1. Schematic representation of the transverse bending mode, the lateral bending mode and the elongation mode.

\subsection{General modeling of the hydrodynamic force}

When a microcantilever vibrates in a viscous fluid (gas or liquid), the fluid offers resistance to the motion. The force per unit length, $F_{\text {fluid }}$, which is the consequence of all normal and tangential stresses (hydrodynamic pressure and viscous shear) exerted by the fluid on all the surfaces of the cantilever, can be written in the frequency domain as [9]:

$$
F_{\text {fluid }}=-\left[j \omega g_{1}(x, \omega)-\omega^{2} g_{2}(x, \omega)\right] w(x, \omega)
$$

where $x$ is the longitudinal coordinate, $\omega$ the radial frequency of vibration, $w$ the microcantilever deflection in the case of bending modes or the microcantilever axial displacement in the case of the elongation mode, and $g_{1}$ and $g_{2}$ are functions depending on the fluid properties and the microcantilever cross-sectional geometry. They may be interpreted, respectively, as the distributed damping coefficient of the fluid and the distributed effective fluid mass (per unit length of beam). In general, these quantities are both frequency-dependent and $x$-dependent; however, in what follows $g_{1}$ and $g_{2}$ will be assumed uniform in $x$ as this assumption has been shown to be sufficiently accurate in many cases of practical interest [6]. 
It is common to normalize $F_{\text {fluid }}$ into a dimensionless form called the hydrodynamic function, $\Gamma=\Gamma_{r}+\mathrm{j} \Gamma_{i}$, where [10]

$$
\begin{aligned}
& \Gamma_{r}=\frac{4}{\pi \rho_{f} b^{2}} g_{2} \\
& \Gamma_{i}=\frac{4}{\pi \rho_{f} b^{2}} \frac{g_{1}}{\omega}
\end{aligned}
$$

The hydrodynamic function, $\Gamma$, represents the total hydrodynamic force per unit length normalized by the force per unit length required to excite (at the same amplitude and frequency) a circular cylindrical volume of diameter $b$ and density $\rho_{f}$. The subscripts $r$ and $i$ in Eqs. 2-3 denote the real and imaginary parts of the hydrodynamic function, respectively.

\subsection{Resonant characteristics of the microstructure}

The fluid effects (viscous term $g_{1}$, and inertial term $g_{2}$ ) influence the dynamic response of the beam; in particular, the resonant frequency, $f_{r}$, and the quality factor associated with viscous losses, $Q_{v i s c}$, may be expressed as $[6,10]$

$$
\begin{gathered}
f_{r}=f_{0, v a c} \frac{1}{\sqrt{1+L g_{2} / m}} \sqrt{1-\frac{1}{2 Q^{2}}} \\
Q_{\text {visc }}=\frac{2 \pi \sqrt{1+L g_{2} / m}}{L g_{1} / m} f_{0, v a c}
\end{gathered}
$$

where $f_{0, v a c}$ is the undamped natural frequency of the microcantilever in vacuum, $Q$ is the total quality factor of the cantilever/fluid system (incorporating all losses), $m$ is the total microcantilever mass and $L$ is the microcantilever length.

The above equations illustrate, as stated in the introduction, the fact that in the general case of dynamic mode operation of a microcantilever, the resonant frequency and the quality factor depend on the fluid properties via the terms $g_{1}$ and $g_{2}$. In the following sections, the terms $g_{1}$ and $g_{2}$ will be analyzed for the different vibration modes.

\subsection{Out-of-plane flexural vibrations of thin microcantilevers}

The hydrodynamic function of a transversely vibrating beam of ribbon-like cross-section (i.e., width $b$ much larger than the thickness $h$ ) was previously determined in [11] by using the method of moments to solve for the velocity field in the fluid and in [10] by performing a fit of the numerical results of [11].

A more accurate approximation of the total hydrodynamic force acting on a transversely vibrating microcantilever in liquids should include both the effects of the pressure and shear stress exerted by the fluid on all faces of the beam of finite cross-sectional dimensions. Due to the symmetry of the problem, the hydrodynamic forces acting in the $x$ or $y$ direction on a beam with a rectangular cross-section vibrating transversely will cancel each other out. Only the hydrodynamic forces acting in the $z$ direction will then affect the response of the beam. These forces are the pressure forces acting on the large faces (of dimension $b$ ) and the shear forces acting on the small faces (of dimension $h$ ). It can be assumed that the microcantilever is long enough so that the hydrodynamic force acting on the surface at the unsupported end of the beam is negligible. Recently, results for the hydrodynamic function in the case of transverse bending that accounted for both the beam's aspect ratio $(h / b)$ and the Reynolds number $(\mathrm{Re})$ of the fluid flow have been published [12]. A Taylor series of the analytical function or a fitting of the numerical results can be made, and then the hydrodynamic function $\Gamma$ can be written as

$$
\begin{aligned}
& \Gamma_{r}=a_{0}(b / h)+a_{1}(b / h) \frac{1}{\sqrt{\mathrm{Re}}}+a_{2}(b / h) \frac{1}{\mathrm{Re}} \\
& \Gamma_{i}=b_{0}(b / h)+b_{1}(b / h) \frac{1}{\sqrt{\mathrm{Re}}}+b_{2}(b / h) \frac{1}{\mathrm{Re}}
\end{aligned}
$$

with $a_{0}, a_{1}, a_{2}, b_{0}, b_{1}$ and $b_{2}$ being functions of aspect ratio $b / h$, and the Reynolds number, Re, defined as 


$$
\operatorname{Re} \equiv \frac{\omega b^{2} \rho_{f}}{4 \eta}
$$

For the ribbon-like case, $a_{0}, a_{1}, a_{2}, b_{0}, b_{1}$ and $b_{2}$ have constant numerical values. In [13] a fit of the results of [10] has been made and the obtained numerical values are:

$$
\begin{array}{lll}
a_{0}=1.0553 & a_{1}=3.7997 & a_{2}=0 \\
b_{0}=0 & b_{1}=3.8018 & b_{2}=2.7364
\end{array}
$$

\subsection{In-plane vibrations of thin microcantilevers}

In 1851, Stokes investigated the forces acting on an infinite rigid plane undergoing a sinusoidal, in-plane motion [14]. This is commonly called Stokes's second problem. The fluid forces on a laterally vibrating beam of infinitely thin crosssection can be considered equivalent to those of Stokes's second problem if the beam under investigation is also quite wide relative to the boundary layer thickness of the fluid. In this case, the hydrodynamic force can be expressed as

$$
\Gamma=\frac{4}{\pi \sqrt{2 \mathrm{Re}}}(1+j)
$$

This equation corresponds to the more general form of Eqs. 6-7 for the following values of coefficients:

$$
\begin{array}{lll}
a_{0}=0 & a_{1}=0.9003 & a_{2}=0 \\
b_{0}=0 & b_{1}=0.9003 & b_{2}=0
\end{array}
$$

However, Eq. 10 neglects edge effects, which could be significant for vibrations in which the boundary layer thickness is not negligible relative to width $b$. Furthermore, it also neglects the fluid resistance due to pressure on the thin faces of the beam (those of corresponding dimension $h$ ) in the lateral bending mode case and the fluid resistance due to shear on the same faces in the elongation mode case. These neglected terms may be important for many practical beam geometries. As was the case for out-of-plane vibrations, it can be assumed that the microcantilever is long enough so that the hydrodynamic force acting on the surface at the unsupported end of the beam is negligible.

In the case of lateral bending, more accurate modeling has been studied in $[12,15,16]$ : the work takes into account both of the edge effects, which could be significant for beams whose boundary layer thickness is not much smaller than the width $b$, and the fluid resistance due to pressure on the thin faces of the beam. These results may also be fit to express them in the form of Eqs. 6-7.

In the case of the elongation mode, it is easy to modify Eq. 11 in order to take into account the hydrodynamic force on the thin faces of the beam (of dimension $h$ ):

$$
\begin{array}{lll}
a_{0}=0 & a_{1}=0.9003(1+h / b) & a_{2}=0 \\
b_{0}=0 & b_{1}=0.9003(1+h / b) & b_{2}=0
\end{array}
$$

In this mode, if we want to take into account the edge effects, numerical simulation may be performed and fitted to express them in the form of Eqs. 6-7.

\section{APPLICATION TO CHEMICAL DETECTION IN LIQUID MEDIA}

From Eq. 4, it can be seen that the decrease of the resonant frequency when the microcantilever is immersed in a fluid is essentially due to the mass effect $g_{2}$; this effect can be minimized if the term $g_{2}$ is small in liquid. On the other hand, the strong decrease in the quality factor in liquid is due to the stronger influence of the viscous term $g_{1}$ compared to the (beneficial) influence of the displaced fluid mass (term due to $g_{2}$ ) in Eq. 5 .

In order to limit the decrease of both the resonant frequency and the quality factor upon immersion in liquid media, alternative vibration modes that essentially shear the surrounding fluid rather than exerting normal stress on it have been studied (section 2.4) and tested. Examples of analyses and experimental measurements of the resonant frequency and quality factor associated with the two unconventional modes of lateral bending and axial elongation are presented in what follows. 


\subsection{Case of lateral bending vibrations}

Several cantilevers with widths of 45, 60, 75 and $90 \mu \mathrm{m}$, lengths of $200,400,600,800,1000 \mu \mathrm{m}$ and nominal silicon thicknesses of 5,8,12 and $20 \mu \mathrm{m}$, corresponding to lateral bending resonant frequencies up to about $3 \mathrm{MHz}$, have been characterized in air and water. For operation in the lateral flexural vibration mode, thermal excitation and piezoresistive detection, both based on the use of diffused resistors, have been chosen as the actuating and sensing mechanisms, respectively. The design of the actuation resistors and integrated Wheatstone bridge allows for a more efficient excitation of the in-plane flexural mode than the more classical out-of-plane flexural mode [17].

The modeling of the fluid interaction terms, $g_{1}$ and $g_{2}$, according to Eq. 10 for the lateral mode results in the resonant frequency being approximately proportional to $b / L^{2}$ (where $b$ is the cantilever width and $L$ the cantilever length), the relative resonant frequency shift from air to water being proportional to $L /\left(h b^{1 / 2}\right)$ and the quality factor (in liquid) being proportional to $h b^{1 / 2} / L$ [18-20]. The results of the simple expression for $Q$ give excellent quantitative agreement for the quality factor in water for aspect ratios up to $h / b=0.2$ [18-20]. However, experimental results show that, for microcantilevers having larger width-to-length ratios, the behaviors predicted by this analytical model differ from measurements. To more accurately model microcantilever resonant behavior in viscous fluids and to improve the understanding of lateral-mode sensor performance, a new analytical model has been developed, incorporating both viscous fluid effects and "Timoshenko beam" effects (shear deformation and rotatory inertia) [21-23]. The latter effects become more important at larger $b / L$ ratios, which are the types of geometries that promise larger $Q$ values in the lateral flexural mode according to the analytical formula derived in [18-20]. The results of the Timoshenko beam model [2123], presented in Fig. 2, exhibit a departure from linearity in both resonant frequency and $Q$ for the shorter, "stubbier" specimens, trends that are also seen in the data. Results such as these serve a useful purpose in serving as guides for selecting microcantilever dimensions to maximize quality factors in liquids (in order to have small noise) and to minimize the liquid-induced decrease in resonant frequency as compared to that in air (in order to yield higher sensitivity).
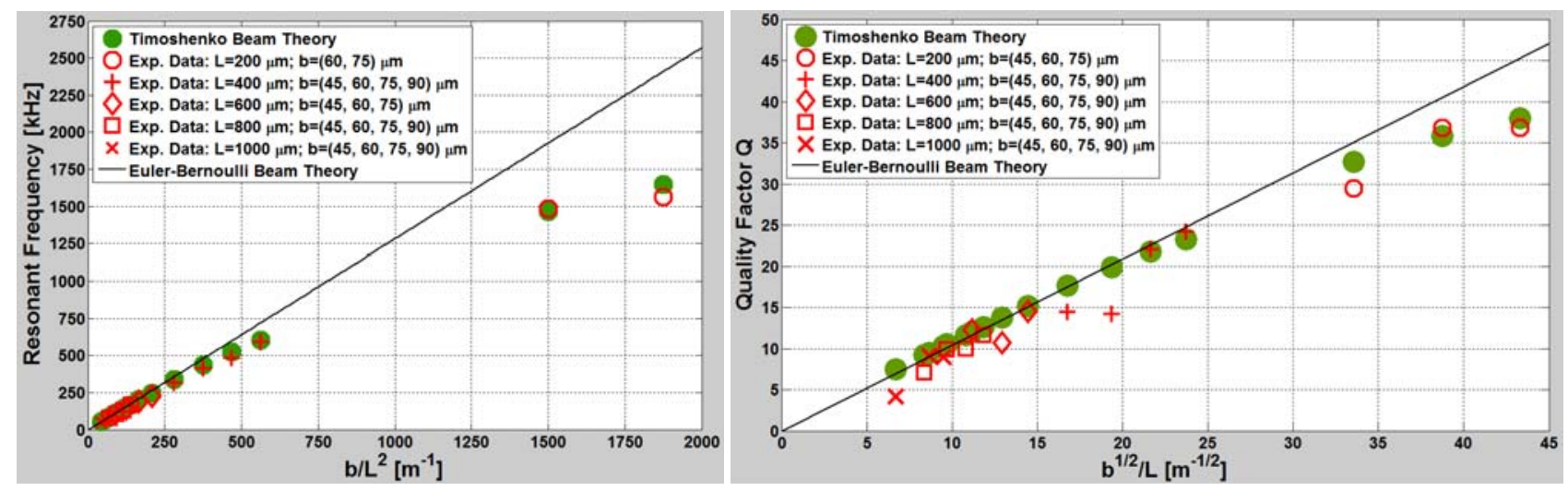

Figure 2. Resonant frequency and quality factor comparison (lateral mode in water, $h=7.02 \mu \mathrm{m}, E=151 \mathrm{GPa}$ ): Timoshenko-based model, Euler-Bernoulli-based model, and experimental data. [20,23]

Analyzing the experimental data shows that (1) for the first lateral flexural mode the microcantilevers exhibit quality factors up to 4300 in air and up to 86 in water (for out-of-plane modes, Q-factors in water usually do not exceed 10 [24]); (2) the resonant frequencies of the in-plane mode are only lowered by $5 \%$ to $10 \%$ in water compared to the values in air (for out-of-plane modes, this reduction is approximately $50 \%$ [6]). These two characteristics of the lateral mode operation are promising for future sensing applications in liquid media.

\subsection{Case of elongation vibrations}

For the same reason as for the lateral bending mode, the longitudinal mode may be of potential interest for sensing applications in liquid media. To assess the characteristics of this mode, self-actuated resonant microcantilevers based on a thick-film piezoelectric layer sandwiched between two electrodes have been processed using a sacrificial layer process [25]. The screen-printed microcantilevers comprising $\mathrm{Au} / \mathrm{PZT} / \mathrm{Au}$ layers were partially released from an alumina substrate and tested [26]. 
Using an impedance analyzer (HP4194A), frequency spectra have been measured in air and in various other fluids with viscosities ranging from 1.5 to $500 \mathrm{cP}$ for a piezoelectric cantilever $\left(8 \times 2 \times 0.1 \mathrm{~mm}^{3}\right)$ with $7 \mu \mathrm{m}$ thick gold electrodes (Fig. 3). Even though the quality factor of 353 in air is relatively low (probably due to internal mechanical losses and piezoelectric losses), the quality factors in liquids are significantly higher compared to those of classical out-of-plane modes. The piezoelectric cantilever exhibits a decrease of the quality factor from 353 to 101 when the cantilever is brought from air to dodecane (with $1.5 \mathrm{cP}$ viscosity -- on the order of that of water, $1 \mathrm{cP}$ ). At the same time, a decrease of resonant frequency of the first longitudinal mode of only $1.8 \%(68.8$ to $67.6 \mathrm{kHz})$ is observed when the cantilever is brought from air to docedane $(1.5 \mathrm{cP})$.

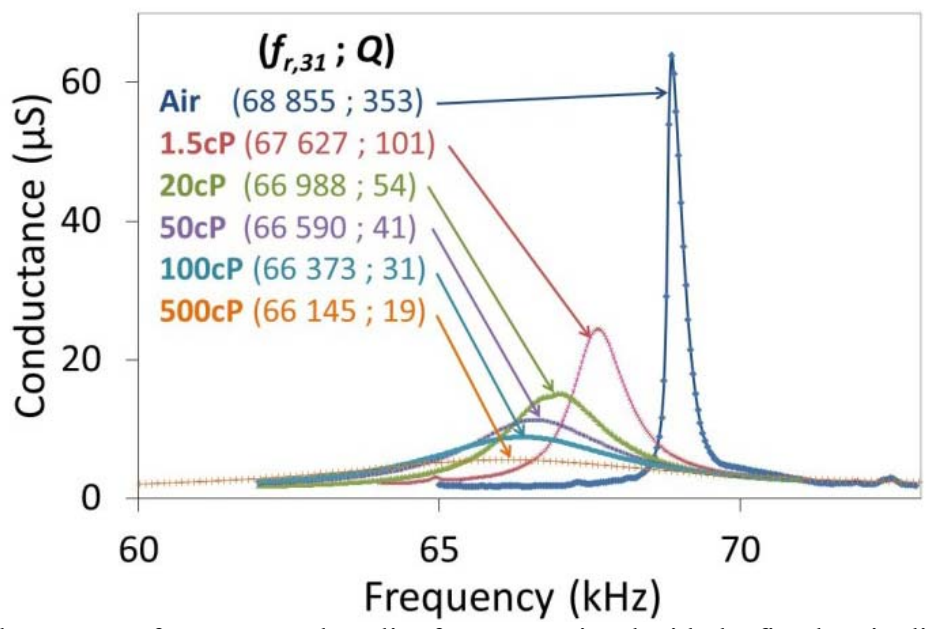

Figure 3. Effects of liquid on the resonant frequency and quality factor associated with the first longitudinal mode of a $8 \times 2 \times 0.1 \mathrm{~mm}^{3}$ PZT cantilever

As was the case with lateral-mode silicon cantilevers having an integrated scheme for actuation and measurement, these thick-film piezoelectric cantilevers actuated in the longitudinal mode are promising in terms of sensitivity and limit of detection for (bio)chemical detection in liquid media.

\section{APPLICATION TO MEASUREMENT OF FLUID RHEOLOGICAL PROPERTIES}

Using the frequency spectra of microcantilevers vibrating in fluids and the equations of section 2, it is possible to extract the mechanical properties of fluids. Different methods have been developed using the transverse bending mode [27]. The first of these permits the determination of both the mass density and viscosity of the fluid at the resonant frequency of the microcantilever in the fluid [28]; the second enables one to extract the same properties over a frequency range that includes the in-fluid resonant frequency [29]; two other methods are based on the same principle, but with the assumption that the fluid's mass density is known. These latter methods allow for the determination of the real and imaginary parts of the fluid's shear modulus, which characterize both the elasticity and viscosity of the fluid [30]. In this section we will focus on presenting a more detailed description of the second method (estimation of mass density and viscosity over a frequency range).

Using the amplitude and phase frequency characteristics of the transverse-bending microcantilever, it is possible to determine the value of the hydrodynamic terms, $g_{1}$ and $g_{2}$, at each measurement frequency.

Then, based on Eqs. 2-3 and 6-9, two analytical equations can be used to obtain the numerical values of both the fluid viscosity, $\eta$, and the fluid mass density, $\rho_{f}$ at the frequency $f$ :

$$
\rho_{f}=\frac{2 \pi f g_{2}\left(2 a_{0} b_{2}-a_{1} b_{1}\right)+a_{2}\left(g_{1} a_{1}-\sqrt{\left(2 \pi f g_{2} b_{1}-a_{1} g_{1}\right)^{2}+8 \pi f g_{2} g_{1} a_{0} b_{2}}\right)}{\pi^{2} b^{2} f a_{0}\left(a_{0} b_{2}-a_{1} b_{1}\right)}
$$




$$
\left.\eta=\frac{a_{0}\left(2 \pi f g_{2} b_{1}+g_{1} a_{1}-\sqrt{\left(2 \pi f g_{2} b_{1}-a_{1} g_{1}\right)^{2}+8 \pi f g_{2} g_{1} a_{0} b_{2}}\right)^{2}}{\pi\left(a_{0} b_{2}-a_{1} b_{1}\right)\left(2 \pi f g_{2}\left(2 a_{0} b_{2}-a_{1} b_{1}\right)+a_{1}\left(g_{1} a_{1}-\sqrt{\left(2 \pi f g_{2} b_{1}-a_{1} g_{1}\right)^{2}+8 \pi f g_{2} g_{1} a_{0} b_{2}}\right)\right.}\right)
$$

In order to measure the amplitude and phase transfer characteristic of microcantilevers in different fluids, chips with silicon-based microcantilevers of uniform rectangular cross section were provided by LAAS-CNRS of Toulouse, France. A series of microcantilevers, termed LL, LH, and A, having the following lengths and widths were tested: $L_{L L}=2810 \mu \mathrm{m}$, $L_{L H}=1440 \mu \mathrm{m}, L_{A}=500 \mu \mathrm{m}, b_{L L}=100 \mu \mathrm{m}, b_{L H}=285 \mu \mathrm{m}, b_{A}=100 \mu \mathrm{m}$. Due to the use of SOI wafers, the silicon thicknesses of all microcantilevers are the same, $h=20 \mu \mathrm{m}$. The microcantilevers were designed to be electromagnetically actuated [30]. For this purpose, a conducting path was deposited on the top surface of the microcantilever. Vibration amplitude measurements were made using an optical vibrometer that detects the out-of-plane velocity (deflection is deduced from the velocity) using Doppler interferometry (MSA 500 Polytec). An example of the spectra in air, water, and egg albumin (for microcantilever LL) is presented in Fig. 4.

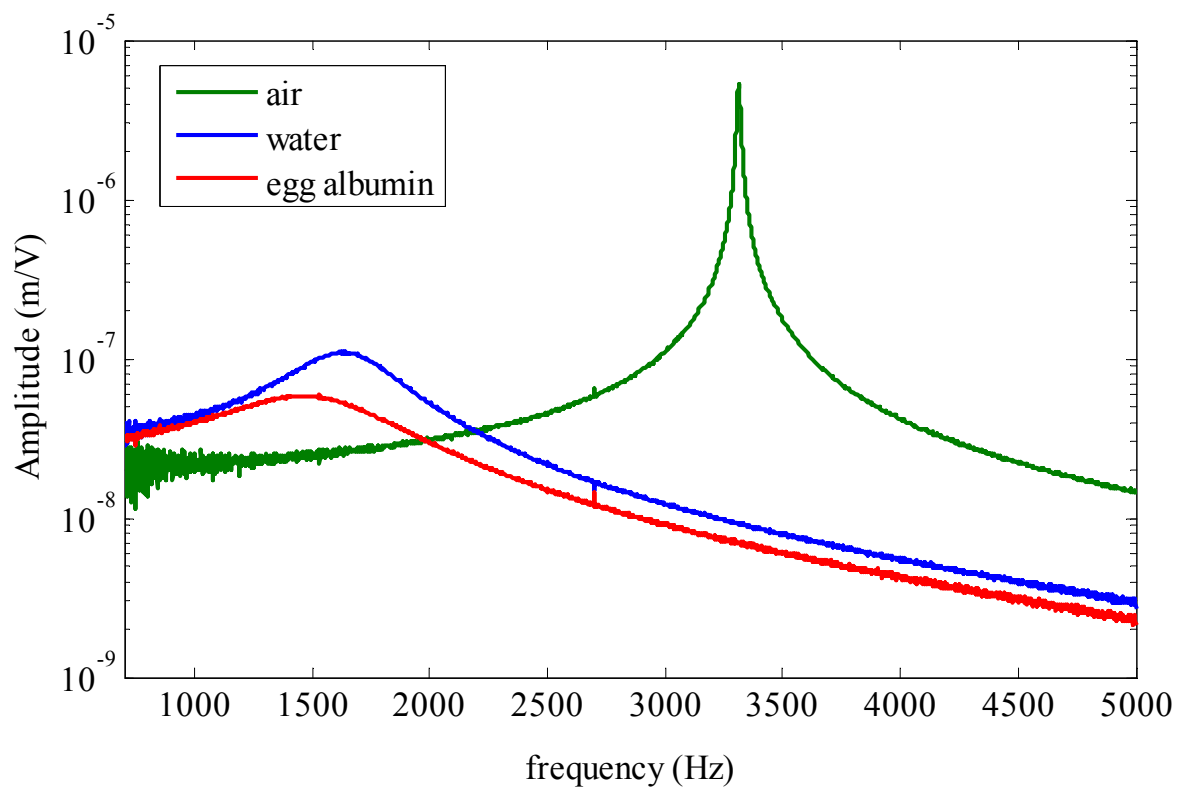

Figure 4. Amplitude of the dynamic motion measured at the free-end of cantilever LL using a laser vibrometer: in air, deionized water and hen egg albumin at $19.4^{\circ} \mathrm{C}$

The extracted fluid properties obtained via measurements of the type shown in Fig. 4, in conjunction with Eqs. 13-14, are presented in Fig. 5. 

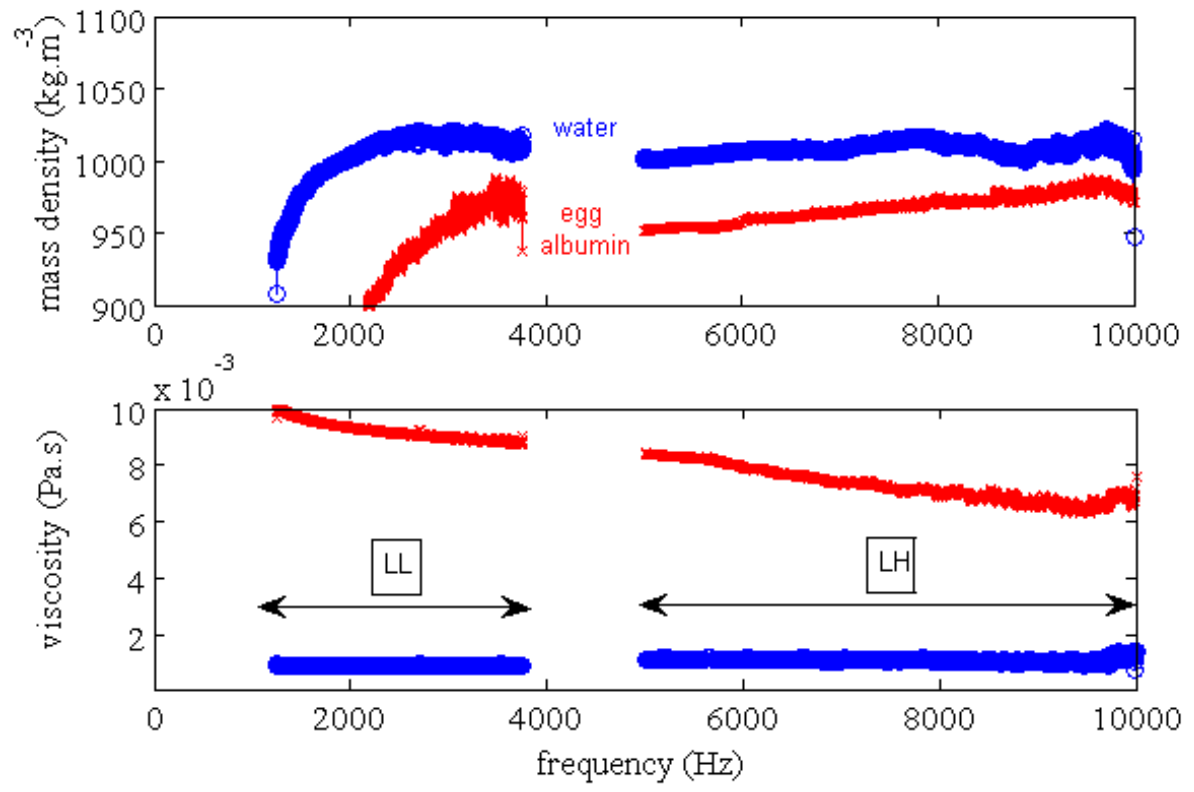

Figure 5. Viscosity and mass density of water (blue circles) and hen egg albumin (red crosses) using the spectrum measurement (amplitude and phase) of two microcantilever geometries, $\mathrm{LL}$ and $\mathrm{LH}$ at $19.4^{\circ} \mathrm{C}$.

The method should correctly evaluate the physical properties of a Newtonian fluid, because with this method the elastic property of the fluid is not taken into account. In Fig. 5, the tests in deionized water show a mass density around $1000 \mathrm{~kg} . \mathrm{m}^{-3}$ (the smaller values at low frequency arise from insufficient accuracy in the estimation of the terms $g_{1}$ and $g_{2}$ which translate to corresponding errors in the mass density estimation) and a constant viscosity around $1 \mathrm{mPa}$.s. Thus we obtain the common properties of water. A non-Newtonian fluid is also measured; the hen albumin has a viscosity that is known to decrease with increasing frequency. This is reflected in the results of Fig. 5. However, in this case the quantitative estimates of both mass density and viscosity are not very accurate because this fluid also possesses elastic properties that have not been accounted for in this method. Nevertheless, the estimated viscosity gives an idea of the frequency-dependent behavior of this complex fluid but, due to its viscoelastic properties, the mass density is not consistent in this case. This problem may be overcome by utilizing one of the methods recently proposed [30], which incorporates the viscoelastic behavior of complex fluids.

Using different microcantilever geometries, the method detailed in this section allows one to measure the fluid viscosity over a large frequency range which is complementary to measurements performed with a classical rheometer, the latter usually being limited to a maximum frequency of $100 \mathrm{~Hz}$.

\section{CONCLUSION}

In a liquid medium, the influence of the hydrodynamic force on the microcantilever's dynamic response is much more pronounced than in a gas medium. Appropriate modeling of this hydrodynamic force, taking into account the particular mode of vibration, is necessary in order to understand the complex interplay among the various system parameters and to achieve the promising potential of these types of devices in numerous applications. In this paper, we have provided an overview of the relevant hydrodynamic force equations for three different modes of vibration (transverse bending, lateral bending and elongation) and presented two different applications for these modeling efforts: (a) the choice of optimal geometry and vibration mode for liquid-phase chemical detection and (b) the determination of the frequency-dependent rheological properties of liquids. 


\section{References}

[1] K.M. Goeders, J.S. Colton and L. A. Bottomley, "Microcantilevers: sensing chemical interactions via mechanical motion," Chemical Reviews, 108, 522-542 (2008).

[2] A. Boisen, S. Dohn, S.S. Keller, S. Schmid and M. Tenje, "Cantilever-like micromechanical sensors," Reports on Progress in Physics, 74, 1-31 (2011).

[3] X. Li and D.W. Lee, "Integrated microcantilevers for high-resolution sensing and probing," Measurement Science and Technology, 23, 022001 (2012).

[4] M. Sepaniak, P. Datskos, N. Lavrik and C. Tipple, "Microcantilever transducers: a new approach in sensor technology," Analytical Chemistry, 74, 568-575 (2002).

[5] Q. Zhu, "Microcantilever sensors in biological and chemical detections," Sensors \& Transducers Journal, 125(2), 1-21 (2011).

[6] J.W.M. Chon, P. Mulvaney and J.E. Sader, "Experimental validation of theoretical models for the frequency response of atomic force microscope cantilever beams immersed in fluids," Journal of Applied Physics, 87, 3978-3988 (2000).

[7] T. Cai, F. Josse, S.M. Heinrich, O. Brand, N. Nigro and I. Dufour, "Resonant characteristics of rectangular microcantilevers vibrating torsionally in viscous liquid media, " IEEE International Frequency Control Symposium, Baltimore, Maryland, USA, 21-24 May 2012, 807-812 (2012).

[8] C. P. Green and J. E. Sader, "Torsional frequency response of cantilever beams immersed in viscous fluids with applications to the atomic force microscope," Journal of Applied Physics, 92(10), 6262 (2002).

[9] R.D. Blevins, Formulas for natural frequency and mode shape, Krieger Publishing Company, Reprint edition 2001.

[10] J. Sader, "Frequency response of cantilever beams immersed in viscous fluids with applications to the atomic force microscope," Journal of Applied Physics, 84, 64-76 (1998).

[11]E. Tuck, "Calculation of unsteady flows due to small motions of cylinders in a viscous fluid," Journal of Engineering Mathematics, 3(1), 29-44 (1969).

[12]D. Brumley, M. Willcox, and J. Sader, "Oscillation of cylinders of rectangular cross section immersed in fluid," Physics of Fluids, 22, 052001 (2010).

[13] A. Maali, C. Hurth, R. Boisgard, C. Jai, T. Cohen-Bouhacina and J-P. Aimé, "Hydrodynamics of oscillating atomic force microscopy cantilevers in viscous fluids," Journal of Applied Physics, 97, 074907 (2005).

[14] G. Stokes, "On the effects of the internal friction of fluids on the motion of pendulums," Transactions of the Cambridge Philosophical Society, 9, 8-106 (1851).

[15] R. Cox, "Theoretical analysis of laterally vibrating microcantilever sensors in a viscous liquid medium," PhD Thesis, Marquette University (2011).

[16]R. Cox, F. Josse, S. Heinrich, O. Brand and I. Dufour, "Characteristics of laterally vibrating resonant microcantilevers in viscous liquid media," Journal of Applied Physics, 111, 014907 (2012).

[17] L.A. Beardslee, A.M. Addous, S. Heinrich, F. Josse, I. Dufour and O. Brand, "Thermal excitation and piezoresistive detection of cantilever in-plane resonance modes for sensing applications," IEEE/ASME Journal of Microelectromechanical Systems, 19, 1015-1017 (2010).

[18] S.M. Heinrich, R. Maharjan, L. Beardslee, O. Brand, I. Dufour and F. Josse, "An analytical model for in-plane flexural vibrations of thin cantilever-based sensors in viscous fluids: applications to chemical sensing in liquids," 7th International Workshop on Nanomechanical Cantilever Sensors, Banff, Canada, 26-28 May 2010, 2 pages (2010).

[19] S.M. Heinrich, R. Maharjan, I. Dufour, F. Josse, L.A. Beardslee, and O. Brand, "An analytical model of a thermally excited microcantilever vibrating laterally in a viscous fluid," Proceedings IEEE Sensors Conference, Waikoloa, Hawaii, November 1-4, 2010, 1399-1404 (2010).

[20] L.A. Beardslee, F. Josse, S.M. Heinrich, I. Dufour and O. Brand, "Geometrical considerations for the design of liquid-phase biochemical sensors using a cantilever's fundamental in-plane mode," Sensors and Actuators B, 164, 7-14 (2012).

[21] J.A. Schultz, "Lateral-mode vibration of microcantilever-based sensors in viscous fluids using Timoshenko beam theory," PhD Thesis, Marquette University (2012).

[22] J. Schultz, S.M. Heinrich, F. Josse, I. Dufour, N.J. Nigro, L.A. Beardslee and O. Brand, "Timoshenko beam model for lateral vibration of liquid-phase microcantilever-based sensors," 14th International Symposium on MEMS and Nanotechnology, SEM 2013, June 3-6 2013, Lombard, IL, USA (2013). 
[23] J.A. Schultz, S.M. Heinrich, F. Josse, N.J. Nigro, I. Dufour, L.A. Beardslee and Oliver Brand, "Timoshenko beam effects in lateral-mode microcantilever-based sensors in liquid," 10th International Workshop on Nanomechanical Cantilever Sensors, Stanford, USA, 1-3 May 2013, 2 pages (2013).

[24]C. Vancura, Y. Li, J. Lichtenberg, K.U. Kirstein, A. Hierlemann, and F. Josse, "Liquid-phase chemical and biochemical detection using fully integrated magnetically actuated complementary metal oxide semiconductor resonant cantilever sensor systems", Analytical Chemistry, 79, 1646-1654 (2007)

[25] C. Lucat, P. Ginet, C. Castille, H. Debéda and F. Ménil, "Microsystems elements based on free-standing thickfilms made with a new sacrificial layer process," Microelectronics Reliability, 48 (6), 872-875 (2008).

[26] R. Lakhmi, H Debéda, C Castille, I Dufour, M. Maglione and C Lucat, "Use of in-plane longitudinal vibrations of screen-printed PZT cantilevers for applications in liquid media," European Meeting on Ferroelectricity. June 26 - July 22011 Bordeaux, France, (2011).

[27] I. Dufour, A. Maali, Y. Amarouchene, C. Ayela, B. Caillard, A. Darwiche, M. Guirardel, H. Kellay, E. Lemaire, F. Mathieu, C. Pellet, D. Saya, M. Youssry, L. Nicu and A. Colin, "The microcantilever: a versatile tool for measuring the rheological properties of complex fluids," Journal of Sensors, 2012, 719898 (2012).

[28] M. Youssry, N. Belmiloud, B. Caillard, C. Ayela, C. Pellet and I. Dufour, "A straightforward determination of fluid viscosity and density using microcantilevers: from experimental data to analytical expressions," Sensors and Actuators A, 172, 40-46 (2011).

[29] N. Belmiloud, I. Dufour, A. Colin and L. Nicu, "Rheological behaviour probed by vibrating microcantilevers," Applied Physics Letters, 92(4), 041907 (2008).

[30] M. Youssry, E. Lemaire, B. Caillard, A. Colin and I. Dufour, "On-chip characterization of the viscoelasticity of complex fluids using microcantilevers," Measurement Science and Technology, 23, 125306 (2012). 